\title{
Guanylate Cyclase
}

National Cancer Institute

\section{Source}

National Cancer Institute. Guanylate Cyclase. NCI Thesaurus. Code C16653.

Guanylate cyclase (GC) is an enzyme that converts GTP to the second messenger cyclic GMP (cGMP). There are two forms: plasma membrane GCs that are receptors for hormones, and intracellular soluble GCs that are activated by the gaseous second messenger nitric oxide (NO). EC 4.6.1.2 